\title{
Identifying the spectroelectrochemical characteristics of hematite pho- toanodes for water oxidation
}

\author{
Jifang Zhang, Qiyuan Lin, Zhenlei Wang, Haowen Liu, and Yuegang Zhang* \\ State Key Laboratory of Low-Dimensional Quantum Physics and Department of Physics, Tsinghua University, Beijing \\ 100084, P.R. China. \\ E-mail: yuegang.zhang@tsinghua.edu.cn
}

\begin{abstract}
Achieving efficient solar water splitting using hematite $\left(\alpha-\mathrm{Fe}_{2} \mathrm{O}_{3}\right)$, one of the most promising candidates for photoanodes, requires photogenerated holes to be efficiently used for water oxidation. However, this goal is obstructed by multiple undesirable recombination processes, as well as insufficient fundamental mechanistic understandings of water oxidation kinetics, particularly as to the nature of reaction pathways and possible reaction intermediates. Here we spectroelectrochemically identify some of the most critical interfacial processes which determine the photoelectrocatalytic efficiencies of water oxidation, for hematite films with varied surface properties by tailoring the doping level of titanium. The spectroscopic signals of the processes inactive for water oxidation, including oxidation of intra-gap $\mathrm{Fe}^{2+}$ states and Fermi level pinning, are successfully distinguished from that of the active reaction intermediate, $\mathrm{Fe}(\mathrm{IV})=0$. In addition, our kinetic analyses reveal two water oxidation pathways, of which the direct hole transfer mechanism becomes dominant over the surface states-mediated mechanism when the hematite surface is reconstructed by high levels of titanium dopants.
\end{abstract}

\section{Introduction}

Water oxidation reaction on the photoanode of a photoelectrochemical (PEC) cell limits the overall solar water splitting efficiency due to its poor reaction kinetics. ${ }^{1}$ Exploring the behavior of photogenerated electron-hole pairs, both near and on the electrode-electrolyte interface is key to tackling this problem. In the field of PEC water oxidation, mechanistic studies at hematite photoanodes are undoubtedly under the spotlight that attracts the attention of researchers specialized at diversified characterization methods, ${ }^{2}$ not only because of its candidature as one of the most promising photoanode materials with a small band gap $(2.2 \mathrm{eV})$ and outstanding stability, but also because of its complex multi-electron charge transfer processes that can involve a number of possible reaction routes.

Over the years, significant advances have been made to understand the kinetics of OER at hematite and other transition metal oxide materials, using techniques such as photoelectrochemical impedance spectroscopy (PEIS), intensity modulated photocurrent spectroscopy (IMPS), and transient photocurrent spectroscopy (TPS). ${ }^{3-6}$ While these time/frequency-resolved methods excel at probing the dynamics of charge carriers, they offer limited insights on the chemical nature of the reaction intermediate species. Transient absorption spectroscopy (TAS) and operando X-ray photoelectron/absorption spectroscopy (XPS/XAS) are powerful tools to acquire physical and chemical information of photoelectrodes, but they are instrumentally complicated and less available to basic electrochemical laboratories. ${ }^{7-9}$ To fill this gap, operando spectroelectrochemical (SEC) techniques coupled with ultraviolet-visible-near infrared (UV-vis-NIR) or IR spectrometers have shown great promises. ${ }^{10-16}$ Compared with operando IR spectroscopy that is relatively chemically specific but can only obtain extremely weak signals that belong to the surface species, ${ }^{10,12}$ UV-vis-NIR-based SEC measurements, sometimes referred to as photo-induced absorption spectroscopy, can collect signals usually with better quality. ${ }^{33,17,18}$ The technique also allows relatively simple cell designs compared with IR spectroscopy. ${ }^{10,12,19}$ Furthermore, using transmission detection mode, this technique should be particularly beneficial for studying highly porous electrodes because signals from a significantly larger surface area can be collected. For these reasons, UV-vis-NIR SEC has been gaining popularity.

For hematite, spectra with a peak centered around its band gap $(570 \mathrm{~nm})$ have been frequently reported with this method, but whether this type of signal is active toward OER has been a heavily debated topic. On the one hand, this spectroscopic feature has been associated with the oxidized form of intra-gap states associated with oxygen vacancies by Durrant's group.7,20 The same observation has also been reported for $\mathrm{BiVO}_{4}$ and their theory is corroborated by directly modulating the filling population of these states..$^{21} \mathrm{On}$ the other hand, many have attributed such finding to the $\mathrm{Fe}(\mathrm{IV})=0$ group, which is the intermediate for the rate limiting step of OER. ${ }^{11,13}$ This assignment has been confirmed by the appearance of a peak at $898 \mathrm{~cm}^{-1}$ from operando attenuated total reflectance infrared spectroscopy. ${ }^{10}$

It is not only the origins of SEC signals but also the OER mechanism that causes dispute. The more widely known pathway is via a four-step, first-order reaction, where the bottleneck product is $\mathrm{Fe}(\mathrm{IV})=0.16,22$ Nevertheless, reaction 


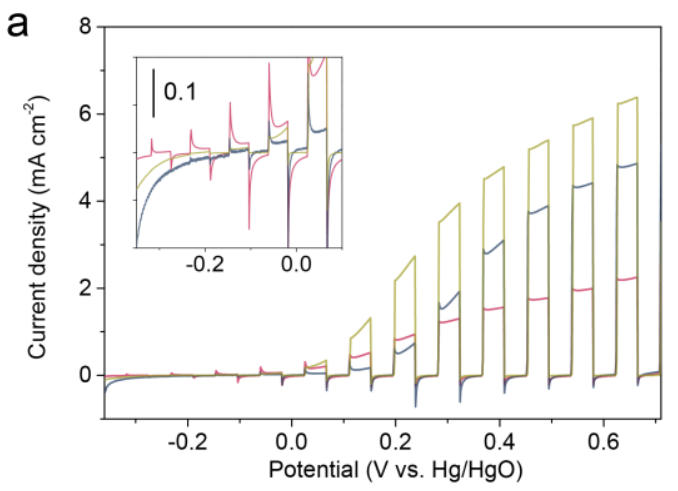

c

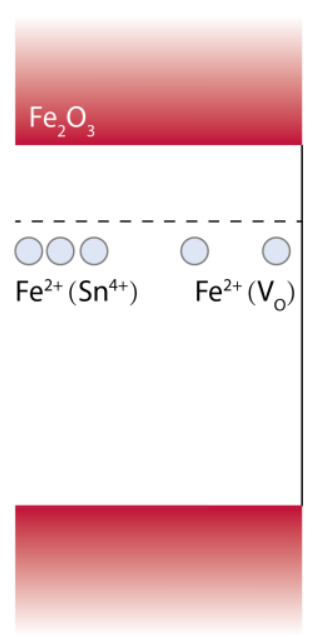

d b
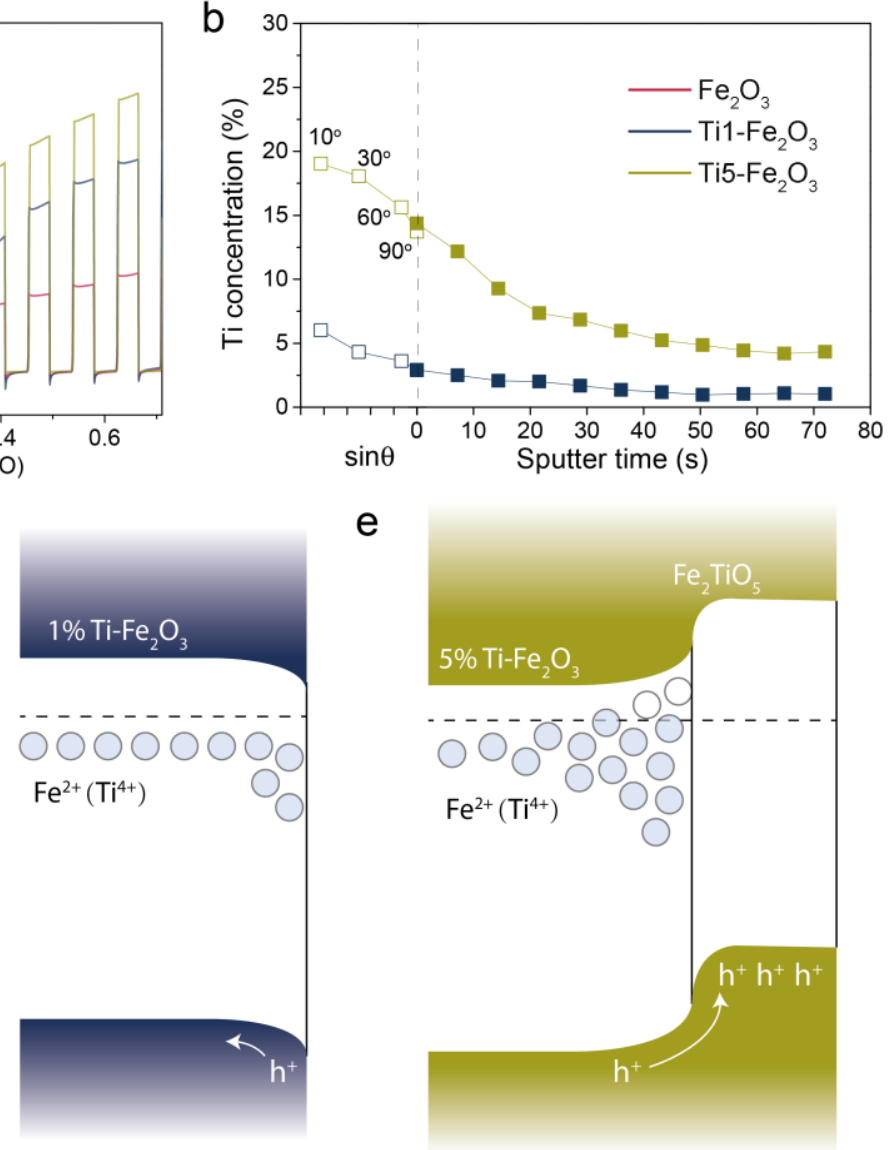

e

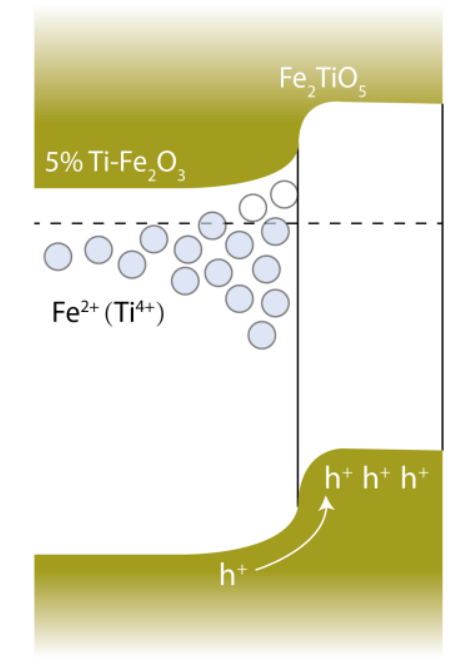

Fig. 1 (a) Current density curves for $\mathrm{Fe}_{2} \mathrm{O}_{3}$ (red), $\mathrm{Ti1}-\mathrm{Fe}_{2} \mathrm{O}_{3}$ (blue) and $\mathrm{Ti5}-\mathrm{Fe}_{2} \mathrm{O}_{3}$ (yellow) under chopped illumination (365 nm LED, $70.6 \mathrm{~mW} \mathrm{~cm}^{-2}$ ) in $1 \mathrm{M} \mathrm{KOH}$ electrolyte solution. Inset shows magnified view at low potentials. $0 \mathrm{VHg} / \mathrm{HgO}=0.95 \mathrm{~V}_{\text {RHE. }}$. Color scheme applies to all following figures. (b) Angle-resolved $\left(10^{\circ}, 30^{\circ}, 60^{\circ}\right.$, and $\left.90^{\circ}\right)$ and depth profiling XPS measurements of Ti doped samples. Each sputtering step corresponds to $1 \mathrm{~nm}$ equivalent of $\mathrm{SiO}_{2}$. (c-e) (Not to scale) Bulk-surface band alignment of $\mathrm{Fe}_{2} \mathrm{O}_{3}$ (c), $\mathrm{Ti}_{1}-\mathrm{Fe}_{2} \mathrm{O}_{3}$ (d) and $\mathrm{Ti5}-\mathrm{Fe}_{2} \mathrm{O}_{3}$ (e), where intra-gap states are induced by oxygen vacancies for $\mathrm{Fe}_{2} \mathrm{O}_{3}$, but by $\mathrm{Ti}^{4+}$ for doped samples. Hole transfer is favorable at the $\mathrm{Fe}_{2} \mathrm{O}_{3} / \mathrm{Fe}_{2} \mathrm{TiO}_{5}$ heterojunction.

pathways with orders of two and three can also be found in the literature. ${ }^{12,23,24}$ Moreover, evidences supporting direct valence band hole transfer to the electrolyte without the involvement of surface states also exist as a counter argument. ${ }^{8}$ These opposing views therefore underscore the necessity to pursue deeper mechanistic insights at hematite photoanodes.

Herein, a fiber spectrometer is, for the first time to our knowledge, used to obtain in-situ UV-vis-NIR spectra. Compared with other reports that adopt a conventional spectrometer, it offers rapid data acquisition capabilities (potentially down to milliseconds) without compromising signal quality $(\Delta$ O.D. sensitivity $<0.1 \%$ ), while eliminating the necessity for specially designed test cells (e.g., homemade SEC cells or modifications to cuvettes). ${ }^{11,13,18,19,21}$

Using this setup, we systematically study several hematite photoanodes with controlled levels of Ti doping, and distinguish various types of SEC signals with seemingly contradictory roles, although all of which peak near the bandgap wavelength, by carefully changing illumination conditions and applied potential/current. We find that the main type of OER-inactive signal is featured by a sharp peak that centers at the band-gap wavelength, whereas the OER- active type has a broader band-gap peak as well as non-trivial features on the longer wavelength side. We combine the spectroscopic findings with kinetic analyses to elaborate two pathways of OER, where the transformation from surface states-mediated route to the direct charge transfer route is highlighted.

\section{Results}

PEC responses of Ti-doped hematite photoanodes. Titanium is arguably the most investigated dopant to improve the PEC performance of hematite photoanodes. ${ }^{25-29}$ As an ntype dopant, it enhances the concentration of electrons, and conceivably the photocurrent density at sufficiently high bias potentials. Nevertheless, the performance at lower potentials seems to differ across the literature: the photocurrent onset potential $\left(E_{\text {on }}\right)$ can be either delayed or improved according to works by others. ${ }^{25-29}$ Here we discover that both scenarios are possible by tailoring the doping concentration of Ti to nominally $1 \%$ and $5 \%$ in the same type of hematite photoanode (denoted as $\mathrm{Ti} 1-\mathrm{Fe}_{2} \mathrm{O}_{3}$ and $\mathrm{Ti} 5-\mathrm{Fe}_{2} \mathrm{O}_{3}$, respectively; undoped sample denoted as $\mathrm{Fe}_{2} \mathrm{O}_{3}$ ). Our $\alpha-\mathrm{Fe}_{2} \mathrm{O}_{3}$ films are prepared from a facile solution-based method (see Methods for detailed preparation procedures), 

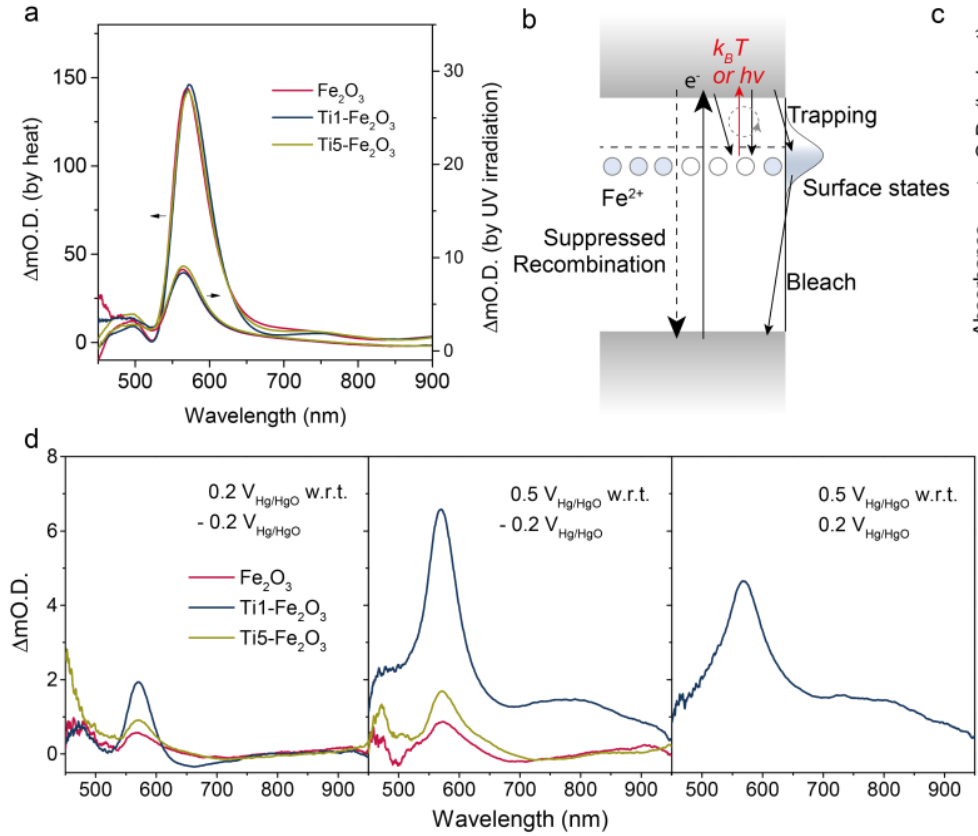

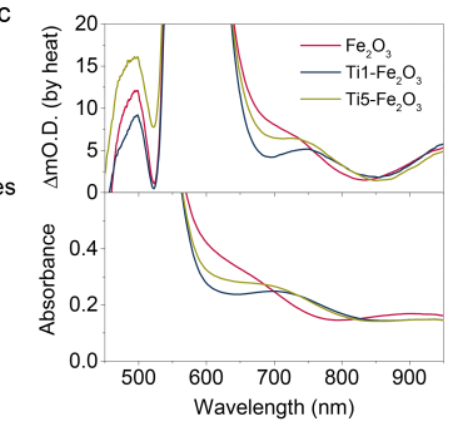

e

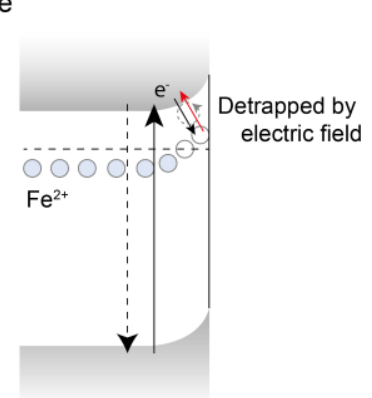

Fig. 2 (a) Difference spectra w.r.t. ground-state absorption of hematite films in the air upon thermal excitation (left axis) and irradiation of $365 \mathrm{~nm}, 70.6 \mathrm{~mW} \mathrm{~cm}^{-2}$ LED light (right axis). (b) Schematic illustration of electronic excitation and relaxation processes in hematite films with intra-gap states with heat and light excitation. Spectroscopic processes in the situation of Fermi level pinning is also shown. (c) Top: Magnified view of (a) under thermal stimulation; bottom: ground-state absorbance spectra. (d) Difference absorption spectra measured at $0.2 \mathrm{~V}_{\mathrm{Hg} / \mathrm{HgO}}$ w.r.t. - $0.2 \mathrm{~V}_{\mathrm{Hg} / \mathrm{HgO}}$ (left), $0.5 \mathrm{~V} \mathrm{Hg} / \mathrm{HgO}$ W.r.t. - $0.2 \mathrm{~V} \mathrm{Hg} / \mathrm{HgO}$ (center), and $0.5 \mathrm{~V}_{\mathrm{Hg} / \mathrm{Hg} 0}$ w.r.t. $0.2 \mathrm{~V}_{\mathrm{Hg} / \mathrm{Hg} 0}$ (right) in the electrochemical cell. (e) Schematic illustration of near-surface electronic excitation and relaxation processes in hematite films at moderately anodic potentials, where some of the intra-gap states inside the space charge layer are ionized electrochemically.

the phases of which are confirmed to be $\alpha-\mathrm{Fe}_{2} \mathrm{O}_{3}$ by X-ray diffraction (Supplementary Fig. 1).

Fig. 1a shows the $J-V$ curve of our hematite films with chopped illumination. One of the causes for the delayed $E_{\text {on }}$ for Ti1- $\mathrm{Fe}_{2} \mathrm{O}_{3}$ has been attributed to the positively shifted flat band potential $\left(E_{\mathrm{fb}}\right),{ }^{26}$ which agrees with our MottSchottky analysis (Supplementary Note 1) when comparing the $E_{\mathrm{fb}}$ of $\mathrm{Fe}_{2} \mathrm{O}_{3} \quad(-0.47 \mathrm{~V} \mathrm{Hg} / \mathrm{HgO})$ with $\mathrm{Ti}_{1}-\mathrm{Fe}_{2} \mathrm{O}_{3}$ $(-0.33 \mathrm{VHg} / \mathrm{HgO})$. Nevertheless, this explanation cannot account for the cathodically shifted $E_{\text {on }}$ for $\mathrm{Ti} 5-\mathrm{Fe}_{2} \mathrm{O}_{3}$ relative to $\mathrm{Ti} 1-\mathrm{Fe}_{2} \mathrm{O}_{3}$, as they both have the same $E_{\mathrm{fb}}$. The lowered onset potential upon Ti doping has been noticed for long but the cause was revealed more recently by Monllor-Satoca et al., who suggested the formation of $\mathrm{Fe}_{2} \mathrm{TiO}_{5}$ near the surface. ${ }^{27,28}$ New phase requires a high dopant concentration to form, as is the case for several publications that generally use over $5 \% .{ }^{27-29} \mathrm{Fe}_{2} \mathrm{TiO}_{5}$ can form a heterojunction with $\mathrm{Fe}_{2} \mathrm{O}_{3}$ that favors hole transport toward the electrode-electrolyte interface due to its higher valence band edge position. ${ }^{28,30,31}$ We will see later in detail how the advent of $\mathrm{Fe}_{2} \mathrm{TiO}_{5}$ restores $E_{\text {on }}$

In the higher potential region, the photocurrent densities $(\mathrm{ph})$ of $\mathrm{Ti} 1-\mathrm{Fe}_{2} \mathrm{O}_{3}$ and $\mathrm{Ti} 5-\mathrm{Fe}_{2} \mathrm{O}_{3}$ are both significantly enhanced due to the higher conductivity as a result of elevated majority charge carrier density $\left(N_{\mathrm{d}}\right)$. For Ti1- $\mathrm{Fe}_{2} \mathrm{O}_{3}$ and Ti5$\mathrm{Fe}_{2} \mathrm{O}_{3}$, Mott-Schottky analysis gives comparable $N_{\mathrm{d}}$ values of $1.88 \times 10^{21}$ and $2.15 \times 10^{21} \mathrm{~cm}^{-3}$, respectively, as opposed to $1.13 \times 10^{20} \mathrm{~cm}^{-3}$ for $\mathrm{Fe}_{2} \mathrm{O}_{3}$; nevertheless, their high-potential $J_{\mathrm{ph}}$ are 2.2 and 2.8 times higher than $\mathrm{Fe}_{2} \mathrm{O}_{3}$, respectively. This discrepancy of the increment of $N_{\mathrm{d}}(14 \%)$ versus increment of $J_{\mathrm{ph}}(27 \%)$ comparing $\mathrm{Ti} 1-\mathrm{Fe}_{2} \mathrm{O}_{3}$ and $\mathrm{Ti} 5$ $\mathrm{Fe}_{2} \mathrm{O}_{3}$ is again a hint of qualitatively altered surface chemistry for OER upon heavy doping at $5 \%$.

Indeed, our angle-resolved and depth-resolved X-ray photoelectron spectroscopy (XPS) measurements show strong gradients of Ti near the surface (Fig. 1b), in agreement with previous findings using $\mathrm{Ti}$ and other $\mathrm{n}$-type dopants. ${ }^{32-34}$ Normal un-sputtered surface XPS results give Ti concentrations of around three times of bulk level and even higher when measured at angles, e.g., $6.0 \%$ and $19.0 \%$ for Ti1- $\mathrm{Fe}_{2} \mathrm{O}_{3}$ and $\mathrm{Ti} 5-\mathrm{Fe}_{2} \mathrm{O}_{3}$ at $10^{\circ}$, respectively. These numbers imply a high probability of phase segregation for Ti5$\mathrm{Fe}_{2} \mathrm{O}_{3}$. An apparent shift of Ti $2 \mathrm{p}$ binding energy from 458.0 ( $\mathrm{Ti} 1-\mathrm{Fe}_{2} \mathrm{O}_{3}$ ) to $458.3 \mathrm{eV}\left(\mathrm{Ti} 5-\mathrm{Fe}_{2} \mathrm{O}_{3}\right)$ further verifies the existence of $\mathrm{Fe}_{2} \mathrm{TiO}_{5}$ (Supplementary Fig. 2). ${ }^{30}$ No peaks, on the other hand, can be assigned to $\mathrm{TiO}_{2}$. It is also worth pointing out that the Ti gradients only arise within about a few nanometers from the surface. Therefore, the formation of $\mathrm{Fe}_{2} \mathrm{TiO}_{5}$ must be extremely limited close to the surface.

Based on the discussions above, the ex-situ bulk-surface band alignment for our hematite samples can be depicted (Fig. 1c-e). For $\mathrm{Fe}_{2} \mathrm{O}_{3}$, intra-gap $\mathrm{Fe}^{2+}$ states might exist as a result of oxygen vacancies. ${ }^{7}$ Introduction of $n$-type dopants such as Ti and Sn is known to elevate the Fermi level of hematite and remove oxygen vacancies, while introducing the $\mathrm{Fe}^{2+}$ states too. 32,35 The gradient doping near the surface, and the concomitant upshifted local Fermi levels would therefore cause a downward band bending. As such, a more 


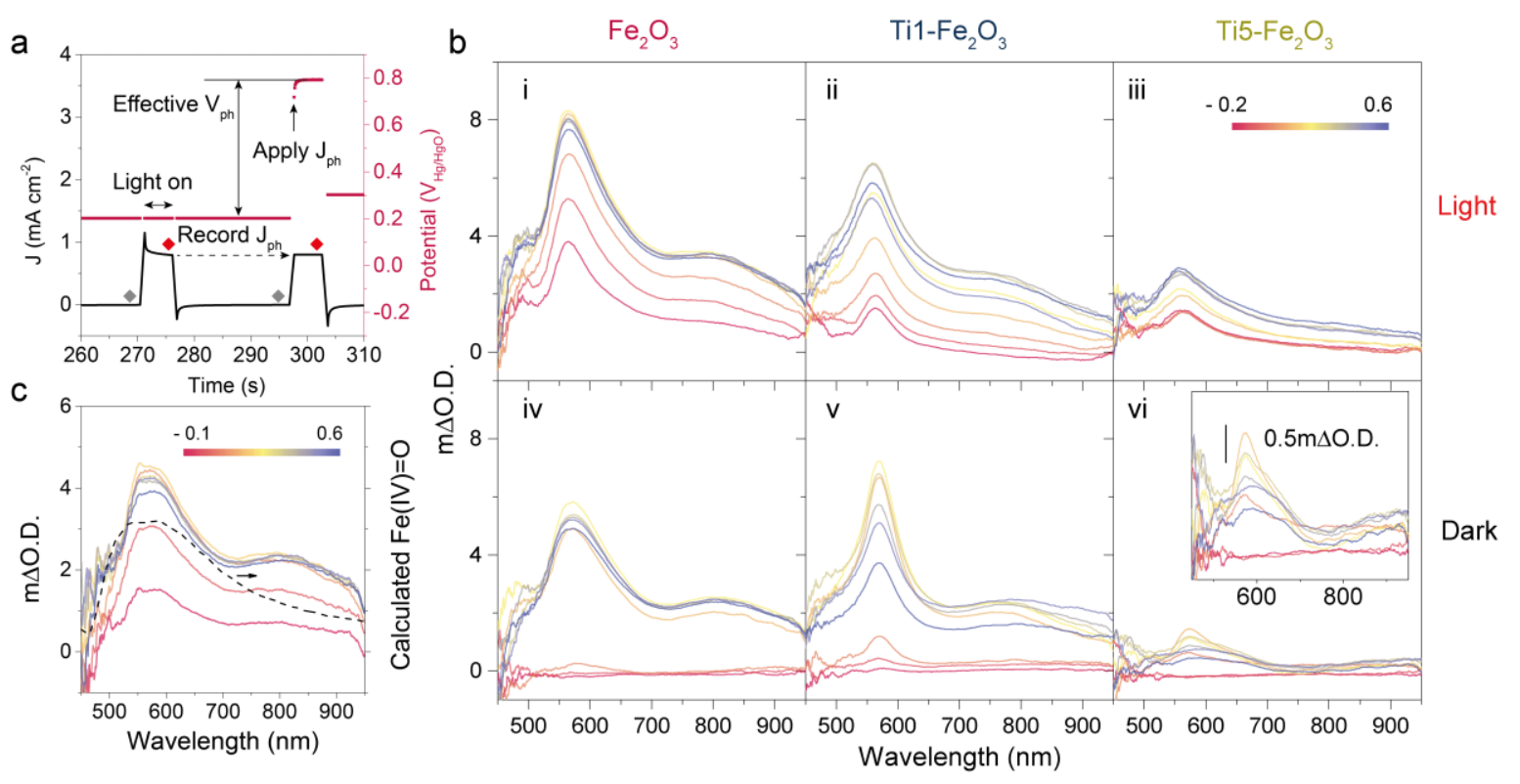

Fig. 3 (a) Potential (red dotted curves) and current density (solid black curve) profiles of $\mathrm{Fe}_{2} \mathrm{O}_{3}$ recorded during a typical stage $\left(0.2 \mathrm{~V}_{\mathrm{Hg} / \mathrm{Hg}}\right)$ for SEC measurements. Grey diamonds and red diamonds indicate points when SEC spectra under reference and OER conditions are taken, respectively. (b) Direct comparison of SEC signals for OER under light (top row) and in the dark (bottom row, with corresponding equivalent OER currents applied as described in the main text) at reference potentials from - $0.2 \mathrm{VHg} / \mathrm{HgO}$ (red) to $0.6 \mathrm{VHg} / \mathrm{Hgo}$ (blue) at a step of $0.1 \mathrm{~V}$ for $\mathrm{Fe}_{2} \mathrm{O}_{3}$ (left column), $\mathrm{Ti}_{1}-\mathrm{Fe}_{2} \mathrm{O}_{3}$ (center column) and $\mathrm{Ti}_{5}-\mathrm{Fe}_{2} \mathrm{O}_{3}$ (right column). (c) Spectra of the main OER intermediate species under illumination calculated by subtracting the - $0.2 \mathrm{VHg} / \mathrm{HgO}$ spectrum from all higher potential spectra in (b, i) for $\mathrm{Fe}_{2} \mathrm{O}_{3}$. For comparison, dashed black curve shows the calculated $\mathrm{Fe}(\mathrm{IV})=0$ spectrum relative to $\mathrm{Fe}(\mathrm{III})-\mathrm{OH}$ by Snir et al. ${ }^{36}$

anodic applied potential is needed to flatten the bands, causing shifts in $E_{\mathrm{fb}}$. The negative effect of this downward bending is substantially eliminated when an $\alpha-\mathrm{Fe}_{2} \mathrm{O}_{3} / \mathrm{Fe}_{2} \mathrm{TiO}_{5}$ heterojunction is formed. To further support our speculation and make it meaningful to compare the three samples, we have ruled out other possible differences in bulk film properties among the three samples by confirming that different PEC performances do not arise from differences in morphology, thickness, and light absorption (Supplementary Fig. 3 and 4). The distinct bulk and surface properties of these three samples of different doping levels will prove to be essential for our SEC studies.

Ex-situ spectroscopic responses. The spectroscopic responses of the samples are first examined in the air with external stimuli of heat and light to see changes in light absorption. Difference spectra are obtained with respect to their ground-state absorption spectra (Fig. 2a). First, the samples are heated by a hot air gun in the dark. As temperature increases (up to roughly $80^{\circ} \mathrm{C}$ ), a peak located at about $570 \mathrm{~nm}$ that matches the band gap grows rapidly. Minor shoulders extending to around $800 \mathrm{~nm}$ can also be seen. In a similar study recently reported on $\mathrm{BiVO}_{4}$, the localized band gap absorption was also observed upon thermal excitation, IR excitation and applied potential. ${ }^{21}$ There this feature was correlated to the ionization of intra-gap $\mathrm{V}^{4+}$ states but not explicitly explained spectrally. Here, we propose that it represents suppressed radiative bulk recombination as opposed to the common perception of extra absorption.

When electrons in these intra-gap states, i.e., $\mathrm{Fe}^{2+}$, induced by oxygen vacancies, polarons, or dopants are thermally excited into the conduction band, these states become empty (Fig. 2b). The photo-generated electrons injected into the conduction band by the light source for the spectrometer (a.k.a. probe) subsequently have the additional option of filling in these states instead of recombination, thereby creating a peak in the difference spectrum. This rapid trapping process is competitive against the picosecond bulk recombination process. ${ }^{7}$ The only difference among the three samples is the minor sub-gap absorption shoulders between 570 and $800 \mathrm{~nm}$. As the constant thermal excitation generates an equilibrized concentration of empty states, more electrons can be excited into them by the probe. This minor absorption should in principle trace the density of intra-gap states when heating heavily depletes the electrons in them. Since these intra-gap states are also mapped by ground-state absorption spectra, it becomes readily understandable that there is a high resemblance of sub-gap spectral shapes measured under stimulated and unstimulated conditions (Fig. 2c).

It should also be mentioned that while $\mathrm{Fe}_{2} \mathrm{O}_{3}$ is not intentionally doped, it also shows pre-edge absorption. This is likely caused predominantly by $\mathrm{Fe}^{2+}$ states induced by unintentional Sn diffusion from the fluorine-doped tin oxide (FT0) substrate (Supplementary Fig. 5) instead of those generated by oxygen vacancies. Interestingly, the location of the peak maximum gradually shifts from $564 \mathrm{~nm}$ at low intensities $(<10 \mathrm{~m} \Delta$ O.D.) to $569 \mathrm{~nm}$ at high intensities ( $>100 \mathrm{~m} \Delta$ O.D., see Supplementary Fig. N2). Unfortunately, the cause of this shift is not entirely clear but we provide some speculations in Supplementary Note 2.

Also shown in Fig. 2a are the difference spectra of samples upon irradiation $\left(365 \mathrm{~nm}, 70.6 \mathrm{~mW} \mathrm{~cm}^{-2}\right.$, denoted 
Table 1 Summary of three main types of SEC signals for $\mathrm{Fe}_{2} \mathrm{O}_{3}$ photoanodes in this work

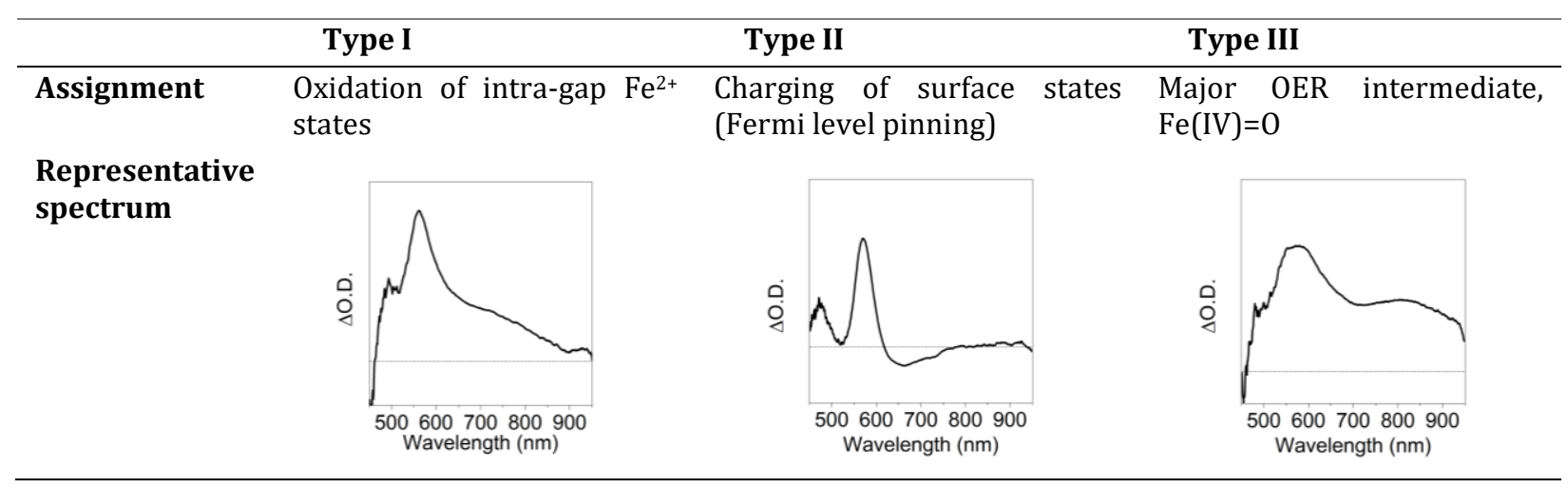

as Type I), which highly resemble the responses of thermal excitation but are more than one order of magnitude weaker.

In this case, a large proportion of the photons are used for supra-gap excitation, thus making the ionization process much less efficient. Consistent with thermal excitation, the peaks observed here are centered around $564 \mathrm{~nm}$ instead of $570 \mathrm{~nm}$ at such low intensities.

SEC measurements. The samples are then moved into the electrochemical cell to investigate their SEC responses at varied bias potentials in the dark. External bias could manipulate the filling population of intra-gap states inside the space charge layer, as well as the surface states, if any, through the changes in band bending. The potential is first set to $-0.2 \mathrm{~V}_{\mathrm{Hg} / \mathrm{HgO}}$ as the referential point and increased stepwise until $0.5 \mathrm{~V}_{\mathrm{Hg} / \mathrm{HgO}}$ (a point sufficiently ahead of the dark water oxidation onsets for all three samples, linear sweep voltammetric plots shown in Supplementary Fig. 6), during which a difference spectrum is collected at each potential stage w.r.t. the $-0.2 \mathrm{~V}_{\mathrm{Hg} / \mathrm{HgO}}$ spectrum (Difference spectra for all steps are given in Supplementary Fig. 7). Below $0.2 \mathrm{~V}_{\mathrm{Hg} / \mathrm{HgO}}, \mathrm{Fe}_{2} \mathrm{O}_{3}$ and $\mathrm{Ti} 5-\mathrm{Fe}_{2} \mathrm{O}_{3}$ show a small absorption peak at $570 \mathrm{~nm}$ but interestingly also negative signals (bleach) between 570 and $800 \mathrm{~nm}$. The bleach has previously been recorded in other reports but left undiscussed.7,20,21 Comparatively, these characteristics are more visible for $\mathrm{Ti1}-\mathrm{Fe}_{2} \mathrm{O}_{3}$ (Spectra with such overall shape are denoted as Type II). Their main peaks both have a full width at half maximum of ca. $50 \mathrm{~nm}$, equal to those generated exsitu by heat or light. By contrast, their intensities considerably lower because these processes are surface sensitive. It should be mentioned that while the three samples have comparable magnitudes of $\mathrm{Fe}^{2+}$ signal outside the cell (Fig. 2a), the low intensity for $\mathrm{Fe}_{2} \mathrm{O}_{3}$ under bias indicates that $\mathrm{Sn}$ diffusion cannot reach the surface due to the short annealing duration of 5 min (inset of Supplementary Fig. 5). The fact that changing potential can only probe the vicinity of the surface also explains the optical signal of $\mathrm{Ti} 5-\mathrm{Fe}_{2} \mathrm{O}_{3}$ being lower than that of $\mathrm{Ti} 1-\mathrm{Fe}_{2} \mathrm{O}_{3}$ in spite of a higher dopant density - many $\mathrm{Ti}$ atoms participate in forming $\mathrm{Fe}_{2} \mathrm{TiO}_{5}$. Above $0.2 \mathrm{VHg} / \mathrm{HgO}$, Type II signals only grow slightly for $\mathrm{Fe}_{2} \mathrm{O}_{3}$ and $\mathrm{Ti5}-\mathrm{Fe}_{2} \mathrm{O}_{3}$ (Fig. 2c, center). In contrast, background starts to build up for $\mathrm{Ti} 1-\mathrm{Fe}_{2} \mathrm{O}_{3}$. Differentiating the spectra recorded at 0.5 and $0.2 \mathrm{~V}_{\mathrm{Hg} / \mathrm{HgO}}$ gives a shape that matches Type I signal generated ex-situ, although the peak is positioned at $570 \mathrm{~nm}$ (Fig. 2c, right).

The origins of these signals are best exemplified by Ti1$\mathrm{Fe}_{2} \mathrm{O}_{3}$. Below $0.2 \mathrm{VHg} / \mathrm{Hg}$, the development of Type II signal is a result of heavy (or complete) Fermi level pinning. The electrons generated near the surface can be strongly trapped by the high density of surface states (Fig. 2b). This process not only suppresses edge-to-edge recombination as before but also yields the bleach signal due to intra-gap recombination in the absence of any effective stimulation. At higher potentials, Fermi level pinning is subdued when surface states are largely depleted, so band bending is recovered. The bending creates empty $\mathrm{Fe}^{2+}$ inside the space charge region (Fig. 2e). Thanks to the newly formed internal electric field that serves as the stimulation here that constantly de-trap these electrons in $\mathrm{Fe}^{2+}$ states, Type I-like signal is observed. Understanding the responses of these electrochemical processes would prepare us to better identify the SEC characteristics under OER conditions.

A direct comparison of SEC signals of electrodes operating at OER conditions under light and in the dark is helpful to elucidate the identities of spectral characteristics and to mechanistically understand OER on hematite. To achieve this, we measure a spectrum at a selected potential in the dark as the reference point. After switching on the LED lamp (365 $\mathrm{nm}, 70.6 \mathrm{~mW} \mathrm{~cm}^{-2}$ ) and the photocurrent stabilizes, another spectrum is collected, using which we obtain the difference spectrum under light. The net steady-state photocurrent is also taken. Subsequently, the same current is applied in the dark and another difference spectrum is taken w.r.t. the same referential condition. This procedure is repeated for nine potentials from -0.2 to $0.6 \mathrm{~V} \mathrm{Hg} / \mathrm{HgO}$ at a step of $0.1 \mathrm{~V}$. Fig. 3a shows an example of this procedure at $0.2 \mathrm{~V}_{\mathrm{Hg} / \mathrm{HgO}}$, and full $J-t$ and $E$ - $t$ profiles are given in Supplementary Fig. 8. The most interesting discovery here is the transformation of spectral shapes under light (Fig. 3b, i-iii). At - $0.2 \mathrm{~V} \mathrm{Hg} / \mathrm{Hg}$, there is a peak located at $564 \mathrm{~nm}$ with a long falling tail toward higher wavelengths. This shape again matches Type I signal and can be associated with oxidized $\mathrm{Fe}^{2+}$ states by photogenerated holes near the surface, with the possibility of weak contributions from OER intermediates since the photocurrent densities are still extremely low $\left(<0.01 \mathrm{~mA} \mathrm{~cm}^{-2}\right)$. 

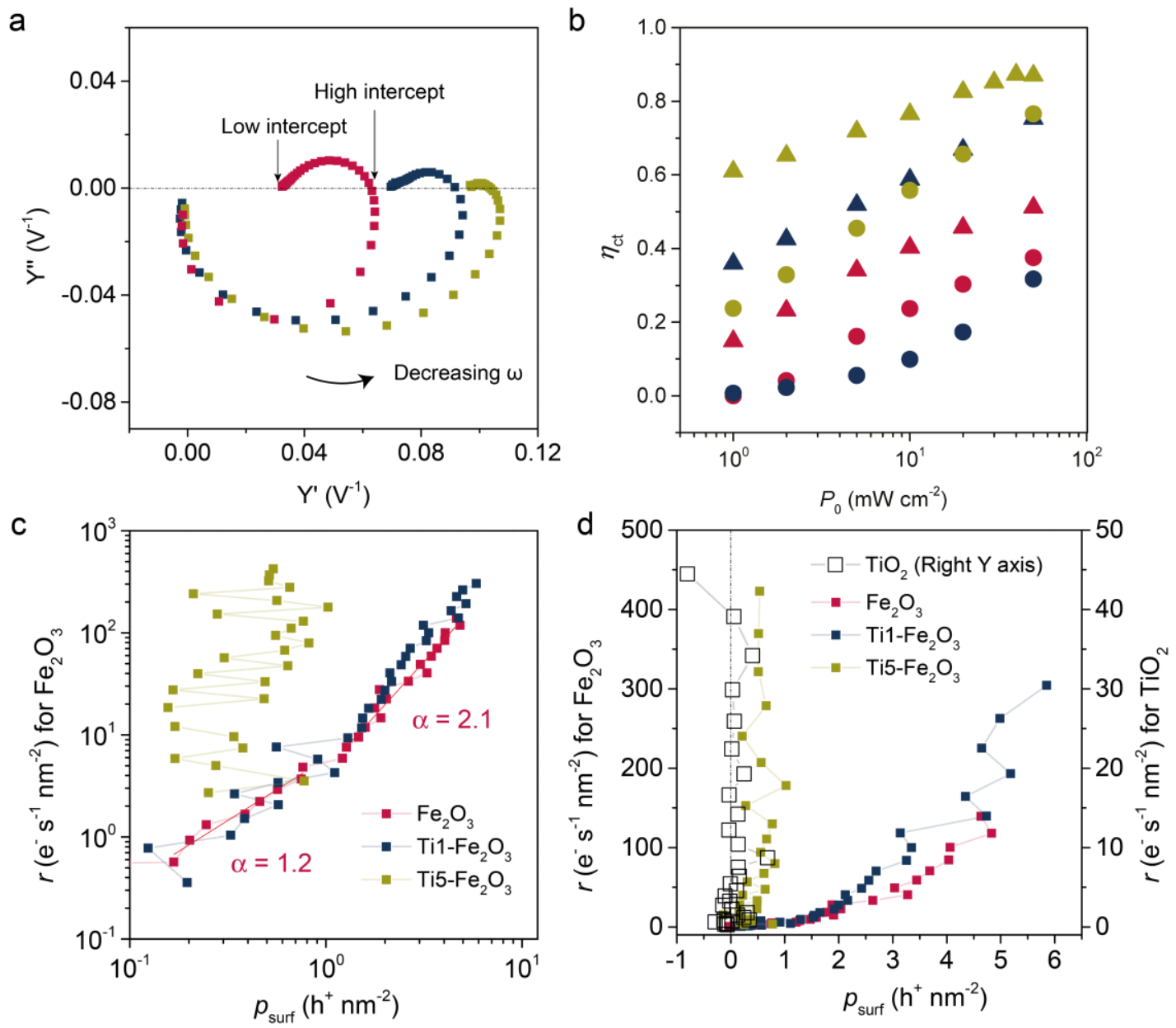

Fig. 4 (a) IMPS results for $\mathrm{Fe}_{2} \mathrm{O}_{3}$ (red), Ti1- $\mathrm{Fe}_{2} \mathrm{O}_{3}$ (blue) and $\mathrm{Ti} 5-\mathrm{Fe}_{2} \mathrm{O}_{3}$ (yellow) measured at $0.6 \mathrm{~V}_{\mathrm{Hg} / \mathrm{HgO}} \mathrm{under} 50 \mathrm{~mW} \mathrm{~cm}^{-2}$ $365 \mathrm{~nm}$ LED light. (b) Charge transfer efficiencies $\left(\eta_{\mathrm{ct}}\right.$ ) calculated from IMPS data at $0.3 \mathrm{~V}_{\mathrm{Hg} / \mathrm{HgO}}$ (circles) and $0.6 \mathrm{~V} \mathrm{~V}_{\mathrm{Hg} / \mathrm{Hg}}$ (triangles) as a function of light intensity for the three samples. (c) Rate law analysis for $\mathrm{Fe}_{2} \mathrm{O}_{3}, \mathrm{Ti}_{1}-\mathrm{Fe}_{2} \mathrm{O}_{3}$ and $\mathrm{Ti} 5-\mathrm{Fe}_{2} \mathrm{O}_{3}$. $\mathrm{Linear}$ fittings at low and high surface charge density regions for $\mathrm{Fe}_{2} \mathrm{O}_{3}$ are shown in solid lines. $r$ is reaction rate and $p_{\text {surf }}$ is surface accumulated charge. (d) Panel (a) plotted in linear scales, with the addition of data for $\mathrm{TiO}_{2}$ (empty black squares, right $Y$ axis) tested under the same conditions.

As soon as potential increases, the growing spectral features begin to deviate from it on top of the original shape. The rise of the intensity of the main peak is accompanied by a broad bump located at $800 \mathrm{~nm}$. Such deviations of characteristics recorded at higher potentials compared to that recorded at $-0.2 \mathrm{~V} \mathrm{Hg} / \mathrm{HgO}$ are also observed for $\mathrm{Ti} 1-\mathrm{Fe}_{2} \mathrm{O}_{3}$ and $\mathrm{Ti}_{5}-\mathrm{Fe}_{2} \mathrm{O}_{3}$. Subtracting the Type I-like spectrum $\left(-0.2 \mathrm{~V}_{\mathrm{Hg} / \mathrm{HgO}}\right.$, light on) from all higher potential spectraunder illumination should give the true optical response of the main OER intermediate (Fig. 3c). As expected, the calculated signals only show changes in magnitude without variations in their characteristics. For $\mathrm{Fe}_{2} \mathrm{O}_{3}$, the signals (denoted as Type III) are similar to those collected under dark OER conditions (Fig. 3b, iv) except a flattened peak apex. The Type III signal with a signature flattened peak highly resonates with the recent computationally obtained spectrum of the $\mathrm{Fe}(\mathrm{IV})=0$ group relative to the neutral $\mathrm{Fe}(\mathrm{III})-\mathrm{OH}$ by Snir et al (dashed lines in Fig. 3c). ${ }^{36}$ The optical signals of OER intermediate for Ti1- $\mathrm{Fe}_{2} \mathrm{O}_{3}$ is identical to $\mathrm{Fe}_{2} \mathrm{O}_{3}$ but those of $\mathrm{Ti} 5-\mathrm{Fe}_{2} \mathrm{O}_{3}$ are slightly different due to the different surface chemical composition (Supplementary Fig. 9). A brief summary of the three main types of SEC signals is included in Table 1.

For SEC signals of $\mathrm{Fe}_{2} \mathrm{O}_{3}$ during dark OER, the extra apex in Fig. $3 \mathrm{~b}$, iv is caused by convolution with Type I signal, which in turn results from a large potential gap straddled (effective photovoltage, $V_{\text {ph }}$ c.f. annotation in Fig. 3 a. See Supplementary Fig. 8 for full profiles). This phenomenon is much more pronounced for $\mathrm{Ti} 1-\mathrm{Fe}_{2} \mathrm{O}_{3}$ at intermediate potentials (Fig. 3b, v) because of the large quantities of $\mathrm{Fe}^{2+}$ available to be oxidized within the corresponding potential windows. In addition, the clear transition from Type I-like signal to Type III-like signal from lower to higher referential potentials for $\mathrm{Ti} 5-\mathrm{Fe}_{2} \mathrm{O}_{3}$ in the dark (inset of Fig. 3b, vi) further attests to this theory.

Below $0.2 \mathrm{~V}_{\mathrm{Hg} / \mathrm{HgO}}$, SEC signals under light build up quickly. But at increasingly higher potentials from around 0.2 up to $0.6 \mathrm{~V} \mathrm{Hg} / \mathrm{HgO}$ (Fig. 3b, i-iii), SEC signals level off whereas photocurrent density continues to grow rapidly. This phenomenon can be simply explained by the initial activation of surface states and then the suppression of surface 
recombination by virtue of recovered band bending. Comparing the spectra across the three samples, the intensities of Type III signals obviously diminish with increasing Ti concentration (Fig. 3c and Supplementary Fig. 9). While it can be argued that it comes from differences in the extinction coefficients of these OER intermediates, it is also likely that OER preferentially proceed via a spectroscopically inactive, intermediate-free pathway. In the following, we provide electrochemical evidences that this could be the direct charge transfer of valence band holes.

Kinetic analysis. To better understand the changes in OER mechanism upon Ti doping, we perform IMPS as a function

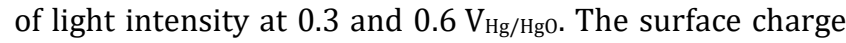
transfer efficiencies $\left(\eta_{\mathrm{ct}}\right)$ are calculated using the ratio of intercepts with the real photocurrent admittance axis as described elsewhere (Fig. 4a). ${ }^{6,37}$ The comparison of the samples at $0.3 \mathrm{~V}_{\mathrm{Hg} / \mathrm{HgO}}$ shows that $\eta_{\mathrm{ct}}$ are poorer for $\mathrm{Ti} 1-\mathrm{Fe}_{2} \mathrm{O}_{3}$ than $\mathrm{Fe}_{2} \mathrm{O}_{3}$, but highest for $\mathrm{Ti} 5-\mathrm{Fe}_{2} \mathrm{O}_{3}$ (Fig. 4b). This proves the adverse effects of the partially offset band bending and the Fermi level pinning, as well as the benefit of charge separation by the $\mathrm{Fe}_{2} \mathrm{O}_{3} / \mathrm{Fe}_{2} \mathrm{TiO}_{5}$ heterojunction. As the surface states are more depleted by the applied potential at $0.6 \mathrm{~V} \mathrm{Hg} / \mathrm{HgO}$, the performance of Ti1- $\mathrm{Fe}_{2} \mathrm{O}_{3}$ quickly catches up. Another important finding that cannot be neglected is the increasing trend of $\eta_{\mathrm{ct}}$ with light intensity for all samples, which has been reported previously with PEIS results. ${ }^{5}$ Likewise, the power conversion efficiency of hematite photoanodes has also been found to improve with higher solar flux concentrations. ${ }^{38}$ This phenomenon has been associated with the extended lifetime of holes, ${ }^{39}$ but further revelations are limited.

From IMPS data, we also calculate the phenomenological charge transfer and surface recombination rate constants based on the pseudo-first order model (Supplementary Fig. 10). ${ }^{37}$ Surprisingly, an anomalous inversion of both parameters occurs for $\mathrm{Ti} 5-\mathrm{Fe}_{2} \mathrm{O}_{3}$ at strong light intensities. Ti1- $\mathrm{Fe}_{2} \mathrm{O}_{3}$ also manifests an inclination for this inversion. Such results again mean the failure of the first-order assumption on hematite photoanodes, and strongly imply a change in OER reaction mechanism.

A population model frequently adopted by Durrant's group, on the other hand, can avoid this assumption and better reveal the true reaction kinetics. ${ }^{16,24,40}$ This method plots reaction rate $(r)$ from current density as a function of surface accumulated charge concentration ( $\left.p_{\text {surf }}\right)$ from cathodic current transient:

$$
r=k_{\mathrm{OER}} \times p_{\text {surf }}^{\alpha}
$$

The reaction order $(\alpha)$ and rate constant (kOER) can be extracted from the slope and the intercept of the double logarithmic plot, respectively. To accurately describe the OER reaction mechanism, the influence of recombination must be eliminated. ${ }^{16}$ In Fig. 3b, we have already seen plateaued SEC signals while photocurrent density keeps increasing. However, our samples show $\eta_{\text {ct }}$ less than unity even at $0.6 \mathrm{VHg} / \mathrm{HgO}$, so it must be considered for $p_{\text {surf }}$ calculation (see Supplementary Fig. 11 for fitted functions of $\eta_{\mathrm{ct}}$ and calculation of $p_{\text {surf). }}$

Fig. 4c shows the rate law analysis for hematite photoanodes at controlled light intensities from 0.2 to $70.6 \mathrm{~mW}$ $\mathrm{cm}^{-2}(365 \mathrm{~nm})$ at a fixed potential of $0.6 \mathrm{~V} \mathrm{Hg} / \mathrm{HgO}$ (Full sets of
SEC spectra in Supplementary Fig. 12). Here the reaction order of $\mathrm{Fe}_{2} \mathrm{O}_{3}$ transforms from 1.2 to 2.1, in good agreement with Zhang's finding. ${ }^{41}$ Accordingly, OER should first involve individual $\mathrm{Fe}(\mathrm{IV})=0$ groups $(\alpha=1)$ and bridged dual $\mathrm{Fe}(\mathrm{IV})=0$ sites $(\alpha=2)$ at low and high hole densities, respectively. The OER kinetics of Ti1- $\mathrm{Fe}_{2} \mathrm{O}_{3}$ is close to that of $\mathrm{Fe}_{2} \mathrm{O}_{3}$, implying a similar surface chemistry. This is also consistent with their similarities in Type III SEC signal shapes (Supplementary Fig. 9). In stark contrast, the high fluctuation of the low levels of surface charge densities for $\mathrm{Ti} 5-\mathrm{Fe}_{2} \mathrm{O}_{3}$ does not allow for a significant rate law fitting. When Fig. 4c is plotted linearly (Fig. 4d), its reaction rate shoots up. This situation more typically applies to a $\mathrm{TiO}_{2}$ photoanode, where photocurrent density increases as a function of light intensity with virtually no accumulation of surface charges. Since $\mathrm{TiO}_{2}$ has very low valence band edge, it is expected to behave more like an ideal semiconductor and undergo direct valence band hole transfer. Therefore, our electrochemical results once more point to the existence of a direct charge transfer regime that accounts for the contribution not observed spectroscopically. Direct charge transfer has also been verified by ambient-pressure operando XPS results. ${ }^{8}$ Shavorskiy et al. examined the relatively ideal undoped and Ti-doped hematite, which did not have light-induced Fermi level pinning effect during OER, meaning there was no surface charge accumulation and photovoltage. Surface passivation of ultrathin $\mathrm{Al}_{2} \mathrm{O}_{3}$ has been demonstrated to strongly suppress SEC signals. Nevertheless, the photocurrent response is insignificantly affected, which further suggests the effectiveness of direct charge transfer for OER. ${ }^{11}$

We believe that such a significant mechanistic change occurring toward high Ti doping levels originates from the intrinsic deficiency of surface states on $\mathrm{Fe}_{2} \mathrm{TiO}_{5}$ over a wide potential window. When it is spontaneously formed simply by introducing dopant source (without recalcination or chemical treatments) on the surface of hematite, especially with poor surface chemistry $\left(E_{\text {on }}>1.0 \mathrm{~V}_{\mathrm{RHE}}\right)$, the $E_{\text {on }}$ can be effectively reduced by about $0.2 \mathrm{~V} .^{27,29}$ Such lack of surface states would therefore facilitate direct hole transfer, thus explaining the low SEC intensities of $\mathrm{Ti} 5-\mathrm{Fe}_{2} \mathrm{O}_{3}$ during light and dark OER. The absence of light-induced Fermi level pinning also leads to the cathodically shifted photocurrent density curve (Fig. 1a).

\section{Conclusion}

In summary, we have used UV-vis-NIR spectroelectrochemistry to observe the optical responses of Ti-doped hematite photoanodes and identified different types of SEC signals that can be either OER inactive or active. We first assign SEC signals that reflect the oxidation of intra-gap $\mathrm{Fe}^{2+}$ states or surface states. Then we assign those for OER intermediates, e.g., $F e(I V)=0$. The main spectral difference between the two is that the former has narrower and pointier peaks than the latter. We have also discovered that OER on hematite photoanode could take place via direct valence band charge transfer without being detected spectroscopically, by reconstructing its surface with heavy Ti doping. These findings are significant for understanding OER processes not only on hematite but also on other transition metal oxide photoanodes. Our ongoing research will emphasize on further exploring the relationship between the two OER pathways for more efficient water splitting. 


\section{ASSOCIATED CONTENT}

The Supplementary Information is available free of charge at http://pubs.acs.org.

\section{AUTHOR INFORMATION}

\section{Corresponding Author}

Yuegang Zhang - State Key Laboratory of Low-Dimensional Quantum Physics and Department of Physics, Tsinghua University, Beijing 100084, P.R. China. E-mail: yuegang.zhang@tsinghua.edu.cn

\section{Author Contributions}

J.Z. conceived the research idea and designed the experiments. J.Z., Q.L. and Z.W. carried out most of the experiments and analyzed the data. H.L. acquired XRD data. Y.Z. directed the project. J.Z. and Y.Z. wrote and revised the paper. All authors reviewed and commented on the paper.

\section{NOTES}

The authors declare no competing interests.

\section{ACKNOWLEDGMENT}

This work is supported by the National Natural Science Foundation of China (No. U1832218) and in part by the Beijing Advanced Innovation Center for Future Chip (ICFC), as well as Frontier Science Center for Quantum Information. J.Z. acknowledges the financial support from China Postdoctoral Science Foundation. J.Z. is grateful to Dr. Weiqi Wang and Dr. Rong Wang of State Key Laboratory of Tribology (THU) for XPS and SEM measurements, respectively. Instrumental assistance from technicians of Metrohm Autolab and Avantes is greatly appreciated.

\section{REFERENCES}

(1) Sivula, K.; Van De Krol, R. Semiconducting Materials for Photoelectrochemical Energy Conversion. Nat. Rev. Mater. 2016, 1, 15010.

(2) Grave, D. A.; Yatom, N.; Ellis, D. S.; Toroker, M. C.; Rothschild, A. The "Rust" Challenge: On the Correlations between Electronic Structure, Excited State Dynamics, and Photoelectrochemical Performance of Hematite Photoanodes for Solar Water Splitting. Adv. Mater. 2018, 1706577, 1-10.

(3) Klahr, B.; Gimenez, S.; Fabregat-Santiago, F.; Hamann, T.; Bisquert, J. Water Oxidation at Hematite Photoelectrodes: The Role of Surface States. J. Am. Chem. Soc. 2012, 134, 4294-4302.

(4) Thorne, J. E.; Jang, J.-W.; Liu, E. Y.; Wang, D. Understanding the Origin of Photoelectrode Performance Enhancement by Probing Surface Kinetics. Chem. Sci. 2016, 7 (5), 3347-3354.

(5) Upul Wijayantha, K. G.; Saremi-Yarahmadi, S.; Peter, L. M. Kinetics of Oxygen Evolution at $\alpha$-Fe203 Photoanodes: A Study by Photoelectrochemical Impedance Spectroscopy. Phys. Chem. Chem. Phys. 2011, 13 (12), 5264-5270.

(6) Zhang, J.; García-Rodríguez, R.; Cameron, P.; Eslava, S. Role of Cobalt-Iron (Oxy)Hydroxide (CoFeOx) as Oxygen Evolution Catalyst on Hematite Photoanodes. Energy Environ. Sci. 2018, 11 (10), 2972-2984.
(7) Barroso, M.; Pendlebury, S. R.; Cowan, A. J.; Durrant, J. R. Charge Carrier Trapping, Recombination and Transfer in Hematite ( $\alpha$-Fe203) Water Splitting Photoanodes. Chem. Sci. 2013, 4 (7), 2724-2734.

(8) Shavorskiy, A.; Ye, X.; Karslloğlu, O.; Poletayev, A. D.; Hartl, M.; Zegkinoglou, I.; Trotochaud, L.; Nemšák, S.; Schneider, C. M.; Crumlin, E. J.; Axnanda, S.; Liu, Z.; Ross, P. N.; Chueh, W.; Bluhm, H. Direct Mapping of Band Positions in Doped and Undoped Hematite during Photoelectrochemical Water Splitting. J. Phys. Chem. Lett. 2017, 8 (22), 5579-5586.

(9) Deng, J.; Zhang, Q.; Lv, X.; Zhang, D.; Xu, H.; Ma, D.; Zhong, J. Understanding Photoelectrochemical Water Oxidation with XRay Absorption Spectroscopy. ACS Energy Lett. 2020, 5 (3), 975993.

(10) Zandi, O.; Hamann, T. W. Determination of Photoelectrochemical Water Oxidation Intermediates on Haematite Electrode Surfaces Using Operando Infrared Spectroscopy. Nat. Chem. 2016, 8 (8), 778-783.

(11) Klahr, B.; Hamann, T. Water Oxidation on Hematite Photoelectrodes: Insight into the Nature of Surface States through in Situ Spectroelectrochemistry. J. Phys. Chem. C 2014, 118 (19), 10393-10399.

(12) Zhang, Y.; Zhang, H.; Liu, A.; Chen, C.; Song, W.; Zhao, J. Rate-Limiting 0-O Bond Formation Pathways for Water Oxidation on Hematite Photoanode. J. Am. Chem. Soc. 2018, 140 (9), 32643269.

(13) Takashima, T.; Ishikawa, K.; Irie, H. Detection of Intermediate Species in Oxygen Evolution on Hematite Electrodes Using Spectroelectrochemical Measurements. J. Phys. Chem. C 2016, 120 (43), 24827-24834.

(14) Ma, Y.; Kafizas, A.; Pendlebury, S. R.; Le Formal, F.; Durrant, J. R. Photoinduced Absorption Spectroscopy of CoPi on BiV04: The Function of CoPi during Water Oxidation. Adv. Funct. Mater. 2016, 26 (27), 4951-4960.

(15) Cummings, C. Y.; Marken, F.; Peter, L. M.; Upul Wijayantha, K. G.; Tahir, A. A. New Insights into Water Splitting at Mesoporous $\alpha$-Fe 203 Films: A Study by Modulated Transmittance and Impedance Spectroscopies. J. Am. Chem. Soc. 2012, 134 (2), 1228-1234.

(16) Le Formal, F.; Pastor, E.; Tilley, S. D.; Mesa, C. A.; Pendlebury, S. R.; Grätzel, M.; Durrant, J. R. Rate Law Analysis of Water Oxidation on a Hematite Surface. J. Am. Chem. Soc. 2015, 137 (20), 6629-6637.

(17) Moss, B.; Hegner, F. S.; Corby, S.; Selim, S.; Francàs, L.; López, N.; Giménez, S.; Galán-Mascarós, J. R.; Durrant, J. R. Unraveling Charge Transfer in CoFe Prussian Blue Modified BiVO 4 Photoanodes. ACS Energy Lett. 2019, 4 (1), 337-342.

(18) Takashima, T.; Hemmi, S.; Liu, Q.; Irie, H. FacetDependent Activity of Hematite Nanocrystals toward the Oxygen Evolution Reaction. Catal. Sci. Technol. 2020, 10 (11), 3748-3754.

(19) Liu, Y.; Le Formal, F.; Boudoire, F.; Guijarro, N. Hematite Photoanodes for Solar Water Splitting: A Detailed Spectroelectrochemical Analysis on the PH-Dependent Performance. ACS Appl. Energy Mater. 2019, 2 (9), 6825-6833.

(20) Barroso, M.; Mesa, C. A.; Pendlebury, S. R.; Cowan, A. J.; Hisatomi, T.; Sivula, K. Dynamics of Photogenerated Holes in Surface Modified $\alpha$ - Fe 203 Photoanodes for Solar Water Splitting. Proc. Natl. Acad. Sci. U. S. A. 2012, 109, 15640-15645.

(21) Selim, S.; Pastor, E.; García-Tecedor, M.; Morris, M. R.; Francàs, L.; Sachs, M.; Moss, B.; Corby, S.; Mesa, C. A.; Gimenez, S.; Kafizas, A.; Bakulin, A. A.; Durrant, J. R. Impact of Oxygen Vacancy Occupancy on Charge Carrier Dynamics in BiV04 Photoanodes. J. Am. Chem. Soc. 2019, 141 (47), 18791-18798.

(22) Zhang, S.; Leng, W. Questioning the Rate Law in the Analysis of Water Oxidation Catalysis on Haematite Photoanodes. Nature Chemistry. 2020, pp 1097-1098.

(23) Li, J.; Wan, W.; Triana, C. A.; Novotny, Z.; Osterwalder, J.; Erni, R.; Patzke, G. R. Dynamic Role of Cluster Cocatalysts on 
Molecular Photoanodes for Water Oxidation. J. Am. Chem. Soc. 2019, 141 (32), 12839-12848.

(24) Mesa, C. A.; Francàs, L.; Yang, K. R.; Garrido-Barros, P.; Pastor, E.; Ma, Y.; Kafizas, A.; Rosser, T. E.; Mayer, M. T.; Reisner, E.; Grätzel, M.; Batista, V. S.; Durrant, J. R. Multihole Water Oxidation Catalysis on Haematite Photoanodes Revealed by Operando Spectroelectrochemistry and DFT. Nat. Chem. 2020, 12 (1), 82-89.

(25) Zhao, X.; Feng, J.; Chen, S.; Huang, Y.; Sum, T. C.; Chen, Z. New Insight into the Roles of Oxygen Vacancies in Hematite for Solar Water Splitting. Phys. Chem. Chem. Phys. 2017, 19 (2), 10741082.

(26) Wang, Z.; Liu, G.; Ding, C.; Chen, Z.; Zhang, F.; Shi, J.; Li, C. Synergetic Effect of Conjugated $\mathrm{Ni}(\mathrm{OH}) 2 / \mathrm{IrO} 2$ Cocatalyst on Titanium-Doped Hematite Photoanode for Solar Water Splitting. J. Phys. Chem. C 2015, 119 (34), 19607-19612.

(27) Wang, G.; Ling, Y.; Wheeler, D. a.; George, K. E. N.; Horsley, K.; Heske, C.; Zhang, J. Z.; Li, Y. Facile Synthesis of Highly Photoactive $\alpha$-Fe203-Based Films for Water Oxidation. Nano Lett. 2011, 11 (8), 3503-3509.

(28) Monllor-Satoca, D.; Bärtsch, M.; Fàbrega, C.; Genç, A.; Reinhard, S.; Andreu, T.; Arbiol, J.; Niederberger, M.; Morante, J. R. What Do You Do, Titanium? Insight into the Role of Titanium Oxide as a Water Oxidation Promoter in Hematite-Based Photoanodes. Energy Environ. Sci. 2015, 8 (11), 3242-3254.

(29) Zandi, O.; Klahr, B. M.; Hamann, T. W. Highly Photoactive Ti-Doped $\alpha$-Fe203 Thin Film Electrodes: Resurrection of the Dead Layer. Energy Environ. Sci. 2013, 6 (2), 634-642.

(30) Deng, J.; Lv, X.; Zhong, J. Photocharged Fe 2 TiO 5 /Fe 20 3 Photoanode for Enhanced Photoelectrochemical Water Oxidation. J. Phys. Chem. C 2018, 122 (51), 29268-29273.

(31) Bassi, P. S.; Chiam, S. Y.; Gurudayal; Barber, J.; Wong, L. H. Hydrothermal Grown Nanoporous Iron Based Titanate, Fe2TiO5 for Light Driven Water Splitting. ACS Appl. Mater. Interfaces 2014, 6 (24), 22490-22495.

(32) Uekawa, N.; Watanabe, M.; Kaneko, K.; Mizukami, F. Mixed-Valence Formation in Highly Oriented Ti-Doped Iron Oxide Film. J. Chem. Soc. Faraday Trans. 1995, 91 (14), 2161-2166.

(33) Gurudayal; Peter, L. M.; Wong, L. H.; Abdi, F. F. Revealing the Influence of Doping and Surface Treatment on the Surface
Carrier Dynamics in Hematite Nanorod Photoanodes. ACS Appl. Mater. Interfaces 2017, 9 (47), 41265-41272.

(34) Dunn, H. K.; Feckl, J. M.; Müller, A.; Fattakhova-Rohlfing, D.; Morehead, S. G.; Roos, J.; Peter, L. M.; Scheu, C.; Bein, T. Tin Doping Speeds up Hole Transfer during Light-Driven Water Oxidation at Hematite Photoanodes. Phys. Chem. Chem. Phys. 2014, $16,24610-24620$.

(35) Tian, C. M.; Li, W. W.; Lin, Y. M.; Yang, Z. Z.; Wang, L.; Du, Y. G.; Xiao, H. Y.; Qiao, L.; Zhang, J. Y.; Chen, L.; Qi, D. C.; MacManusDriscoll, J. L.; Zhang, K. H. L. Electronic Structure, Optical Properties, and Photoelectrochemical Activity of Sn-Doped Fe203Thin Films. J. Phys. Chem. C 2020, 124 (23), 12548-12558.

(36) Snir, N.; Toroker, M. C. The Operando Optical Spectrum of Hematite during Water Splitting through a GW-BSE Calculation. J. Chem. Theory Comput. 2020, 16 (8), 4857-4864.

(37) Lewrenz, H.-J.; Peter, L. Photoelectrochemical Water Splitting: Materials, Processes and Arhitectures; RSC Publishing, 2013.

(38) Segev, G.; Dotan, H.; Malviya, K. D.; Kay, A.; Mayer, M. T.; Grätzel, M.; Rothschild, A. High Solar Flux Concentration Water Splitting with Hematite ( $\alpha$-Fe203) Photoanodes. Adv. Energy Mater. 2016, 6, 1500817.

(39) Le Formal, F.; Pendlebury, S. R.; Cornuz, M.; Tilley, S. D.; Grätzel, M.; Durrant, J. R.; Formal, F. Le; Pendlebury, S. R.; Cornuz, M.; Tilley, S. D.; Grätzel, M.; Durrant, J. R.; Le Formal, F.; Pendlebury, S. R.; Cornuz, M.; Tilley, S. D.; Grätzel, M.; Durrant, J. R.; Formal, F. Le; Pendlebury, S. R.; Cornuz, M.; Tilley, S. D.; Grätzel, M.; Durrant, J. R. Back Electron - Hole Recombination in Hematite Photoanodes for Water Splitting. J. Am. Chem. Soc. 2014, 136 (6), 2564-2574.

(40) Mesa, C. A.; Steier, L.; Moss, B.; Francàs, L.; Thorne, J. E.; Grätzel, M.; Durrant, J. R. Impact of the Synthesis Route on the Water Oxidation Kinetics of Hematite Photoanodes. J. Phys. Chem. Lett. 2020, 11 (17), 7285-7290.

(41) Li, N.; Wei, W.; Xie, K.; Tan, J.; Zhang, L.; Luo, X.; Yuan, K.; Song, Q.; Li, H.; Shen, C.; Ryan, E. M.; Liu, L.; Wei, B. Suppressing Dendritic Lithium Formation Using Porous Media in Lithium MetalBased Batteries. Nano Lett. 2018, 18 (3), 2067-2073. 